\title{
COMPUTER-AIDED ENGINEERING
}

\section{СИСТЕМЫ АВТОМАТИЗАЦИИ ПРОЕКТИРОВАНИЯ}

\section{УДК 004.4'2}

\author{
А.Е. Ивасев, Ю.А. Лапшов, К.В. Святов
}

\section{ПРОГРАММНОЕ УПРАВЛЕНИЕ БАЗОЙ ОПЫТА ПРОЕКТИРОВЩИКА АВТОМАТИЗИРОВАННЫХ СИСТЕМ ${ }^{1}$}

Ивасев Александр Евгеньевич, окончил факультет информационных систем и технологий Ульяновского государственного технического университета, аспирант УлГТУ. Область научных интересов системы автоматизированного проектирования. [e-mail: alex.ivasev@gmail.com].

Лапшов Юрий Александрович, кандидат технических наук, окончил факультет информационных систем и технологий УлГТУ, доцент кафедры «Вычислительная техника» УлГТУ. Область научных интересов - проектирование автоматизированных систем, интенсивно использующих программное обеспечение. [e-mail: y.lapshov@mail.ru].

Святов Кирилл Валерьевич, кандидат технических наук, доцент, окончил факультет информационных систем и технологий УлГТУ. Декан факультета информационных систем и технологий УлГТУ, заведующий кафедрой “Вычислительная техника» УлГтУ. Область научных интересов - машинное обучение и робототехника. [e-mail: k.svyatov@ulstu.ru].

Аннотация

В статье представлен подход к формированию базы опыта проектной организации, ориентированный на применение его в разработке автоматизированных систем (АC), интенсивно использующих программное обеспечение. Использование предлагаемой базы опыта расширяет потенциал инструментально-моделирующей среды OWnWIQA на рабочем месте члена группы проектировщиков АС. К специфике инструментария относится формирование в процессе выполнения работ по созданию АС модели повторного использования (модели прецедента), которая включается в базу опыта с целью ее дальнейшего использования при развитии АС или создания новых АС из того же семейства.

В статье рассмотрена модель базы знаний проектов, представлен механизм поиска прецедентов, реализованный в среде OWnWIQA, описаны экспериментальные исследования, раскрывающие параметры точности и полноты поиска прецедентов в базе знаний, а также рассмотрены факторы, влияющие на эти показатели для разных пользователей системы.

Работа может быть интересна специалистам в области построения баз знаний и опыта.

Ключевые слова: модель опыта, модель прецедента, проектная задача, управление знаниями, инструментально-технологические средства.

doi: 10.35752/1991-2927-2021-1-63-110-118

1 Исследование проводится при поддержке грантов РФФИ 18-47-730016 р_а и 18-07-00989 А. 


\section{THE SOFTWARE CONTROLLED EXPERIENCE BASE OF A COMPUTER-AIDED SYSTEM DESIGNER}

Aleksandr Evgenevich Ivasev, Postgraduate Student of Ulyanovsk State Technical University; graduated from the Faculty of Information Systems and Technologies of UISTU; the area of his scientific interests relates to computer-aided design systems.e-mail:alex.ivasev@gmail.com.

Iurii Aleksandrovich Lapshov, Candidate of Sciences in Engineering; graduated from the Faculty of Information Systems and Technologies of Ulyanovsk State Technical University; Associate Professor of the Computer Engineering Department; the area of his scientific interests relates to designing the software-intensive automated systems.e-mail: y.lapshov@mail.ru.

Kirill Valerevich Sviatov, Candidate of Sciences in Engineering; graduated from the Faculty of Information Systems and Technologies of Ulyanovsk State Technical University; Dean of the Faculty of Information Systems and Technologies of UISTU; Head of the Computer Engineering Department; the area of his scientific interests relates to machine learning and robotics.e-mail: k.svyatov@ulstu.ru.

\section{Abstract}

The article describes an approach to the creation of an experience base for a software design organization, which is focused on its application in the development of software-intensive automated systems (AS). The use of the proposed experience base extends the potential of the OwnWIQA tool-modeling environment in the workplace of a member of the software design team. The specificity of the toolkit includes the design of a reusable model (precedent model) in the process of work execution on the design of an AS, which is included in the experience base for the purpose of its further use in the development of AS or the creation of a new AS from the same family.

The article describes the knowledge base model of software projects, tools for finding use cases, implemented in the OwnWIQA environment. Experimental studies are described that reveal the parameters of precision and recall for a precedent search in the knowledge base. The factors influencing these indicators for different users of the system are given.

The article may be of interest to specialists in the field of building knowledge and experience bases.

Keywords: experience base, precedent model, project task, knowledge management, instrumental and technological tools.

\section{Введение}

По данным исследований, проводимых Standish Group [1], в 2019 году 89\% всех проектов в области разработки автоматизированных систем (АС), интенсивно использующих программное обеспечение (ПО), заканчивались полным или частичным «провалом» в части сроков, бюджета или качества разрабатываемого по. При этом ключевыми факторами, влияющими на низкую успешность разработок, оказывались следующие:

- отсутствие вовлеченности конечных пользователей ПО;

- неполные требования и спецификации;

- частое изменение требований и спецификаций в процессе разработки;

- отсутствие поддержки со стороны руководства;

- техническая некомпетентность специалистов, осуществляющих разработку.

Стандарт ISO/MEK 9004-2009 содержит спецификацию понятия "успех» в его приложении к проектам и организациям. Успех организации (успех ее проектов) в существенной мере определяется наличием необходимого и востребованного опыта и тем, насколько полно он представлен и широко доступен в коллективной работе [2].
Большое влияние на стабильность разработки и развития АС оказывает профессиональная деятельность проектировщика, т. к. он является автором исходной концепции и у него есть целостное видение проекта [3]. Проектировщик одним из первых приступает к работе над системой и продолжает следить за ней в процессе реализации. Многое зависит от опыта, который есть у проектировщика, и от того, как он им умеет пользоваться. При этом важным аспектом деятельности такого специалиста является использование им специальных баз опыта, которые не являются изолированными и статичными [4]. Данные в базе опыта должны быть актуальными и доступными для изучения другими проектировщиками.

В данной статье проведен анализ задач, которые решает проектировщик, с целью определения того, какие данные полезны в его работе. Также рассмотрено само понятие базы опыта и составляющих ее частей. В работе описан инструментарий, встроенный в OWnWIQA [5], который позволяет извлекать данные из базы опыта проектировщика.

\section{ЗАДАЧИ ПРОЕКТИРОВЩИКА}

В толковом словаре Ушакова проектировщик - это специалист по проектированию [6]. В небольших ком- 
паниях обычно проектировщик - это человек, обладающий наибольшим уровнем владения компетенциями в создании АС. Такой специалист анализирует не только свой опыт, но и опыт всей команды. При этом он должен обладать знаниями технологий и отлично понимать бизнес-процессы [7].

На начальном этапе проектировщику необходимо проанализировать требования технического задания на разработку системы, продумать структуру AC и технологии, которые будут использоваться во время разработки. Неправильно спроектированная архитектура или выбранная технология увеличивают риск временных или финансовых издержек, связанных с невозможностью внедрения новой функциональности. Чтобы проектировщик быстрее и качественнее решал эту задачу, ему нужен набор шаблонов архитектурных решений. Если эти решения уже проверены практикой и есть записи об их эффективности в конкретных условиях, то это увеличивает шанс их уместного применения.

\section{БАЗА ОПЫТА}

База опыта (Experience Base) - это репозиторий, содержащий интегрированный набор проанализированных и «упакованных» моделей опыта, который был использован в предыдущих проектах и доступен по оперативным запросам для повторных использований [8].

Если представление и интеграция единиц индивидуального и коллективного опыта подобны, то в рамках подобия для индивида его взаимодействие как с моделью своего опыта, так и с моделями опыта других индивидов (в рассматриваемом случае членов коллектива), будет осуществляться по единым схемам. В этом случае модель коллективного опыта образует расширение модели индивидуального опыта [9].

В моделировании коллективного опыта, учитывающего специфику семейств AC, для ориентира был взят стандарт Framework for Software Product Line Practice Version 5.0 (FSPLP.V5.0), разработанный в Институте программной инженерии (Software Engineer Institute, SEI) Университета Карнеги-Меллон.

База опыта конкретного проектировщика строится в результате осуществления проекта специализированной $\boldsymbol{A} \boldsymbol{C}^{\boldsymbol{\sigma} O}$, предназначенной для обслуживания деятельности, по разработке семейства $\boldsymbol{A C}$, включая те $\boldsymbol{A C}$, которые реализованы по проектам других специалистов, например, по проектам $\boldsymbol{F}$ и $\boldsymbol{G}$.

Основным источником моделей активов и «местом» их совершенствования являются проекты, разрабатываемые в проектной организации, к которым имеет доступ проектировщик.

Модель очередного потенциального актива $\boldsymbol{M}^{\boldsymbol{F}}\left(\boldsymbol{Z}_{\boldsymbol{i j}}\right)$ (рис. 1), освоенного в проекте $\boldsymbol{F}$, преобразуется в форму $\boldsymbol{M}^{\boldsymbol{E} \boldsymbol{O}}\left(\boldsymbol{Z}_{\boldsymbol{i j}}\right)$, подготовленную для ее повторного применения в других проектах. После того, как модель $\boldsymbol{M}^{\boldsymbol{E} \boldsymbol{O}}\left(\boldsymbol{Z}_{\boldsymbol{i j}}\right)$ подготовлена к повторному ис- пользованию, она помещается в базу опыта и для нее создается каталожная карточка. По запросу из другого проекта $\boldsymbol{G}$, копия модели $\boldsymbol{M}^{\boldsymbol{E} \boldsymbol{O}}\left(\boldsymbol{Z}_{\boldsymbol{i j}}\right)$, найденная с помощью каталога, извлекается из репозитория и используется в этом проекте, если это оказалось полезным. При необходимости модель адаптируется, а, возможно, создается ее измененная версия, модель которой также помещается в базу опыта. Из-за того, что создание и модификация моделей типа $\boldsymbol{M}^{\boldsymbol{Б}}$ осуществляется проектировщиками, которые создавали их прототипы типа $\boldsymbol{M}^{\boldsymbol{I}}$, средства, необходимые для такой работы, размещены на рабочих местах проектировщиков [10].

В изначальном состоянии база опыта содержала в себе только модель проекта $\boldsymbol{F}$.

$$
M_{1}^{E O}\left(Z_{i j}\right)=M^{F}\left(Z_{i j}\right) \text {. }
$$

По завершению проекта $\boldsymbol{G}$ база опыта содержит модели проектов $\boldsymbol{F}$ и $\boldsymbol{G}$.

$$
M_{2}^{\boldsymbol{B O}}\left(Z_{i j}\right)=M^{F}\left(Z_{i j}\right) \cup M^{G}\left(Z_{i j}\right) \text {. }
$$

Из этого следует, что

$$
\begin{aligned}
& \Delta M_{n}^{\boldsymbol{B O}}\left(Z_{i j}\right)=M^{n}\left(Z_{i j}\right) \mid M_{n-1}{ }^{Б O}\left(Z_{i j}\right), \\
& M^{\boldsymbol{B O}}\left(Z_{i j}\right)=\sum_{n=1}^{\infty} \Delta M_{n}^{\boldsymbol{B} O}\left(Z_{i j}\right) .
\end{aligned}
$$

Если модель нового проекта полностью покрыта моделью текущей базы опыта, то после её добавления не произойдет увеличения базы.

В свою очередь модель проекта состоит из всех задач, которые решались на проекте и становятся прецедентами (рис. 1).

$$
\boldsymbol{M}^{\boldsymbol{\Pi}}=\sum_{i=0}^{n} \sum_{j=0}^{m} \boldsymbol{Z}_{i \boldsymbol{j}}
$$

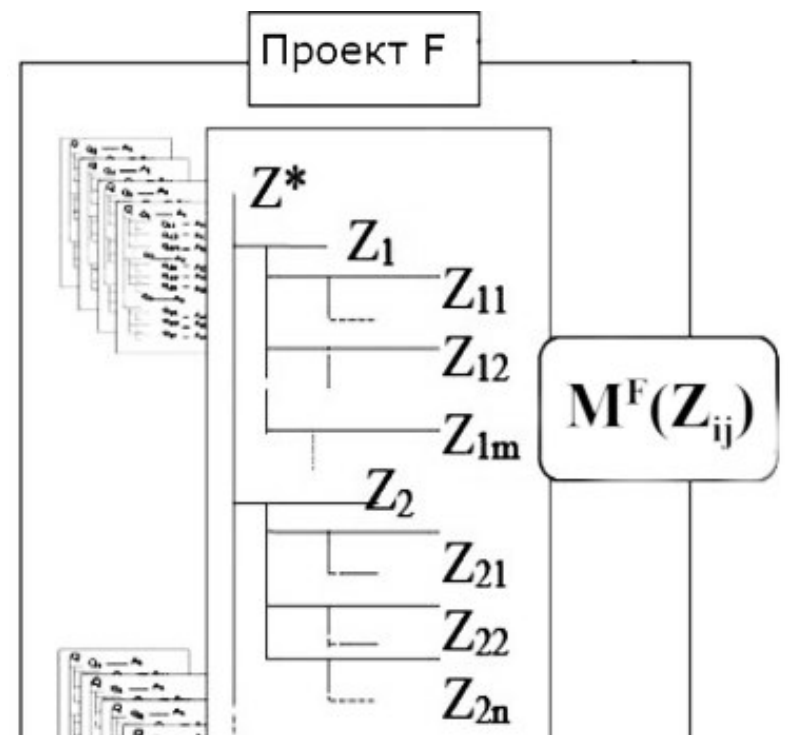

Рис. 1. Иерархия задач в структуре проекта 
Обобщенная схема представления активов в базе опыта приведена на рисунке 2, где отражен и тот факт, что размещение моделей активов осуществляется по разделам, в соответствии с типологией активов, используемой в проектной организации [11].

\section{ФОРМАЛИЗАЦИЯ ОТОБРАЖЕНИЯ ПРЕLЕДЕНТА НА ПАМЯТЬ WIQA}

С позиции рассматриваемого в статье отображения прецедента на память OwnWIQA, прецедент состоит из двух основных частей:

- Задача в терминологии вопросно-ответной среды WIQA;

- Карточка или набор карточек в каталоге среды WIQA для представления метаданных прецедента.

Для формализации будем применять расширенную форму Бэкус-Наура (РБНФ).

\section{Прецедент $=($ Задача, $\{$ Карточка $\})$.}

Для хранения задач в памяти OWnWIQA используется вопросно-ответная память WIQA. В вопросно-ответной (QA) памяти $\boldsymbol{W I Q} \boldsymbol{A}$ содержится набор QA-объектов:

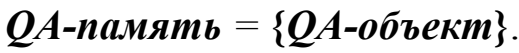

Сам QA-объект представляет собой пару, состоящую из вопроса и ответа, причем ответ может быть пустым или отсутствовать:

\section{QА-объект = Задача $\mid$ Вопрос, «њ», Ответ.}

Поскольку сама память OwnWIQA предполагает отношения вхождения (соответствия) и иерархического подчинения, введём два нетерминала для обозначения этих отношений. Нетерминал «ヶ», будем использовать для обозначения вхождения (соответствия), а нетерминал « $\downarrow$ »-для обозначения иерархического подчинения.

Задача может содержать в себе как подзадачи, так и вопросы:

$$
\begin{aligned}
& \text { Задача }=Z|(Z, « \downarrow »,\{Z\})|(Z, « \downarrow »,\{Q\}) ; \\
& \text { Вопрос }=Q \mid(Q, \ll \downarrow »,\{Q\}) ; \\
& \text { Ответ }=A \mid(A, \ll \downarrow »,\{A\}) .
\end{aligned}
$$

Онтология в среде WIQA представлена в виде набора словарей $\boldsymbol{D}$ :

\section{Онтология $=\{D\}$.}

Каждый словарь включает в себя набор групп понятий $\boldsymbol{G}$ :

$$
\boldsymbol{D}=\{\boldsymbol{G}\}
$$

Каждая группа понятий может включать как вложенные группы понятий, так и сами понятия:

$$
\boldsymbol{G}=\{P\} \mid(G, \ll \downarrow »,\{G\}),
$$

где $\boldsymbol{P}$ - понятие.

Каждое понятие включает в себя его текстовое обозначение, набор отношений к другим понятиям и набор определений:

$$
P=(T,\{R\},\{D\}) .
$$

Здесь нетерминал $\boldsymbol{T}$ представляет собой текстовое обозначение понятия в виде строки, $\boldsymbol{R}$ означает отношение к другому понятию, а $\boldsymbol{D}$ - текстовое определение.

Карточка каталога для использования ее в базе прецедентов представляет собой понятие, у которого текстовое обозначение представляет собой заголовок, а определение содержит $\boldsymbol{X M L}$-описание полей карточки.

\section{Карточка $=\boldsymbol{P}$.}

Определение $\boldsymbol{D}$ для карточки $(\boldsymbol{D} \boldsymbol{c d})$ содержит в себе как минимум следующие поля, представленные в виде XML-тегов: ID задачи, к которой относится эта карточка (Zid), набор ключевых слов $(\boldsymbol{K})$, дата создания $(\boldsymbol{D} \boldsymbol{c})$, дата изменения $(\boldsymbol{D} \boldsymbol{e})$, автор задачи $(\boldsymbol{Z} \boldsymbol{a})$. Кроме того, она может содержать набор дополнительных полей $\boldsymbol{M d}$.

$$
D c d=(Z i d,\{K\}, D c, D e, Z a,\{M d\}) .
$$

Здесь Zid представляет собой целое число, $\boldsymbol{K}, \boldsymbol{Z a}$, $\boldsymbol{M d}$ - строки, $\boldsymbol{D} \boldsymbol{c}, \boldsymbol{D} \boldsymbol{e}$ - даты.

На рисунке 3 представлена модель базы знаний прецедентов с позиции рассматриваемой в данной работе функциональности.

\section{БАЗА ПРЕЦЕДЕНТОВ}

В своей профессиональной и повседневной деятельности человек решает множество задач, начиная от самых простых бытовых, заканчивая сложнейшими профессиональными. Чаще всего схожие между собой 


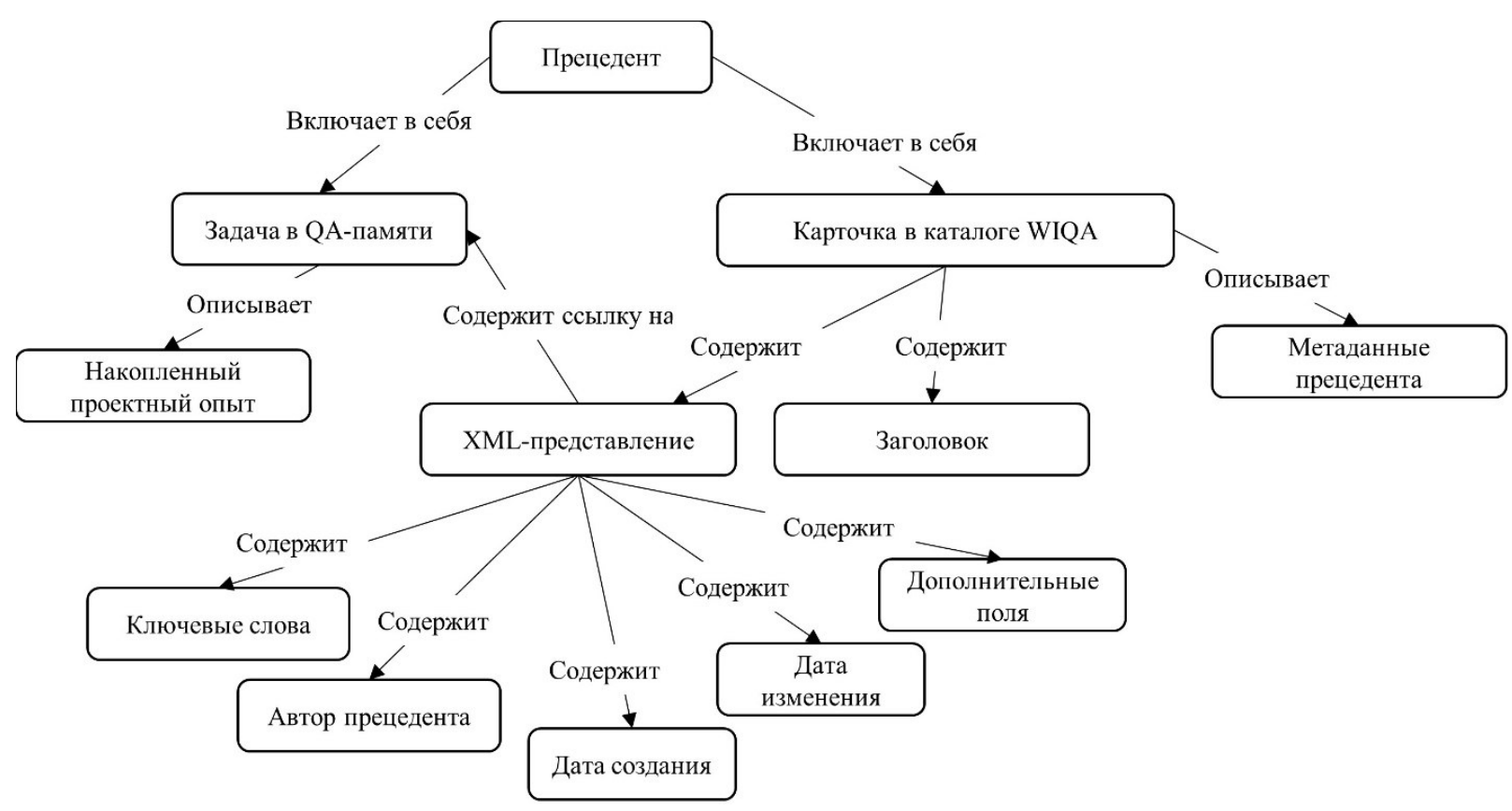

Рис. 3. Модель базы знаний

задачи приходится решать повторно в процессе профессиональной деятельности. При этом информация, необходимая для решения задач, должна быть легко доступна. Всю необходимую информацию запомнить невозможно, поэтому создание базы прецедентов как средства для хранения информации в удобной для чтения и для поиска форме является важным направлением исследований.

Проектировщик и архитектор АС должны иметь сборник основных паттернов, шаблонов решений, необходимых для профессиональной деятельности. Наличие такого сборника важно не только для конкретного проектировщика, но и для всей компании с точки зрения экономии ресурсов на обучение новых сотрудников [12]. Часто полноценное обучение не производится, в результате случаются происшествия, связанные с тем, что работник просто не знает, как правильно себя вести в рабочей ситуации, и это может привести к чрезвычайным ситуациям. И в итоге время и ресурсы, которые компания тратит на устранение последствий, значительно превышают те, которые требуются для создания базы прецедентов.

Практическая реализация базы прецедентов была осуществлена в вопросно-ответной моделирующей среде OWnWIQA, зарекомендовавшей себя на этапе концептуального проектирования АС, интенсивно использующих ПО [5].

Вначале были разработаны схемы взаимодействия проектировщика со средой (диаграммы последовательностей), опи- сывающие два основных процесса. Во-первых, получение решения по заданной задаче (рис. 4). Когда пользователь приступает к выполнению новой задачи, он осуществляет поиск по базе прецедентов. Если до этого аналогичные задачи уже решались, то пользователь найдет прецедент. Из найденного прецедента пользователь получит решение, которое сможет использовать повторно полностью или частично.

Во-вторых, добавление нового прецедента, которое происходит в тот момент, когда в базе не был найден прецедент, в котором решалась аналогичная задача

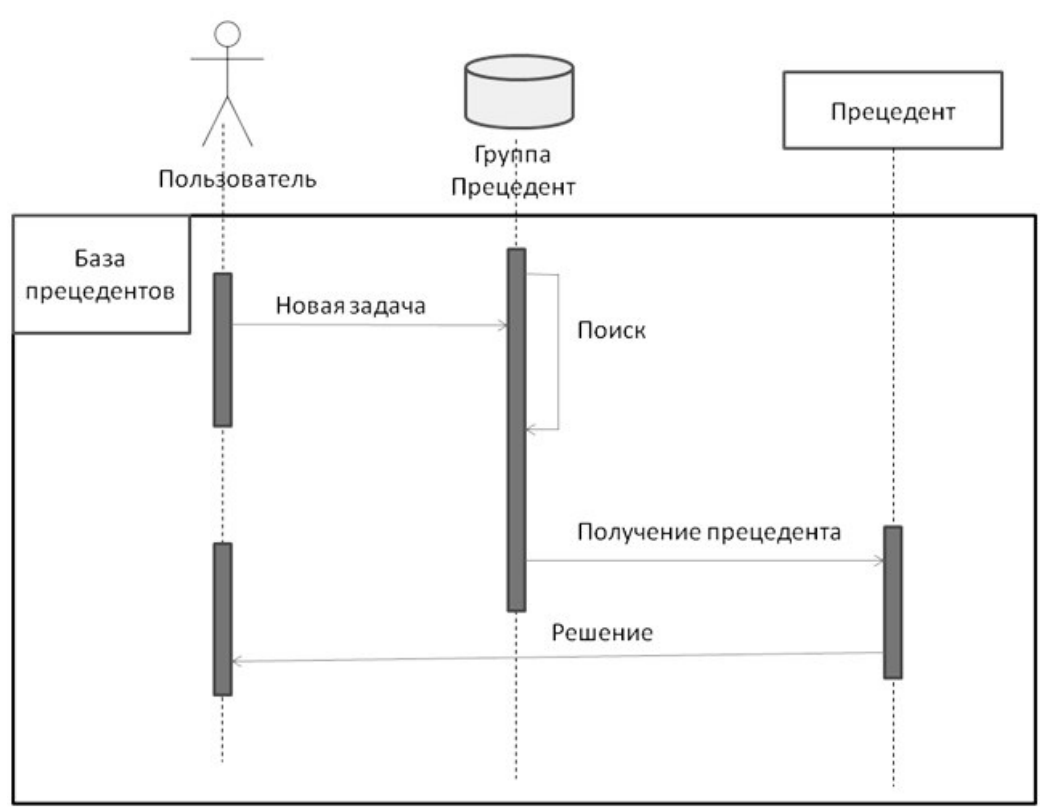

Рис. 4. Оценка. Получение решения по прецедентам 
(рис. 5). Для результатов, полученных в ходе решения новой задачи, будет создана карточка, которая может быть найдена в дальнейшем.

В среде OWnWIQA реализован комплекс программных средств, позволяющих пополнить базу прецедентов как единиц опыта проектировщиков и производить поиск в ней. Для поиска используется окно (рис. 6), в котором слева располагаются поля для ввода данных фильтрации и две кнопки, а справа находится изначально пустая область, в которую после фильтрации загружаются найденные прецеденты.

Фильтрация осуществляется после нажатия на кнопку "Найти» (Рис. 6). Поиск полного совпадения происходит по полю «id». По полям «Автор», «Ключи», «Описание» происходит поиск вхождений строки запроса в соответствующих свойствах. Так же есть возможность фильтрации по двум типам, в которых хранятся значения для полей «дата создания» и «дата изменения» путем указания диапазона значений для фильтрации. После выполнения процедуры поиска в правой части выводится список всех найденных карточек прецедентов (рис. 6).

По выбору элемента в списке происходит открытие диалогового окна (рис. 7), где отображается вся информация из карточки прецедента. В случае, если в карточке указан путь к файлу и он физически есть, то будет выведена кнопка «Файл». После нажатия на кнопку будет открыт файл.

В случае отсутствия нужной карточки или ее неполного описания, проектировщик может добавить новую карточку в каталог или изменить ее, чтобы в дальнейшем иметь возможность воспользоваться накопленным опытом.

Данные карточки хранятся в формате XML-кода в виде текста понятия онтологии. Такой формат хранения данных удобен тем, что набор данных, которые в нем хранятся, легко реструктурировать. Этот формат накладывает свои ограничения на логику поиска данных по диапазону, связанные с трудностями выделения информации, которая повышает эффективность поиска.
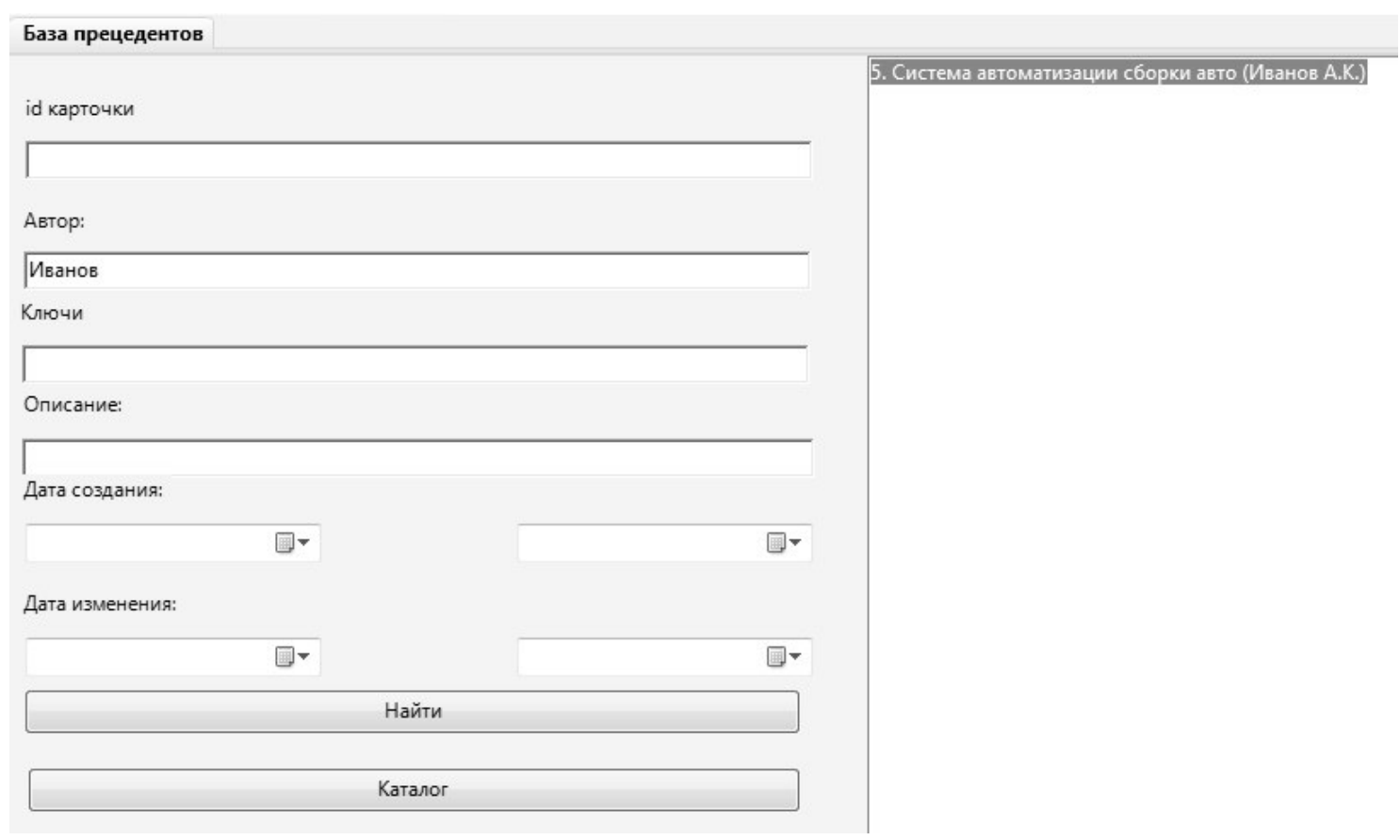

Рис. 6. Результат поиска 
Сейчас поиск реализован в два этапа (рис. 8). В начале происходит поиск карточек по полям: «id», «Автор», «Ключи», «Описание». Вторым этапом осуществляется фильтрация найденных карточек по полям: «дата создания», «дата изменения».

В дальнейшем планируется реализация средств поиска прецедентов с использованием специализированных поисковых движков.

Основной причиной выбора подхода, в котором проектировщик сам заполняет карточку, - это трудности автоматического выделения основных параметров и проведения классификации проектов, из которых получены прецеденты. Автоматизация данного процесса становится возможной после создания объемной выборки карточек в базе прецедентов, проведения их анализа с целью выделения терминов и ключевых характеристик единиц опыта, которые проектировщики считают важными. В дальнейшем планируется реализовать автоматическую предобработку информации об AC на предмет выявления ключевых терминов, описывающих артефакты проектирования, их связей между собой и с артефактами проектирования, а также поиск прецедентов, содержащих такие термины.

\section{ОЦЦЕНКА ЭФФЕКТИВНОСТИ}

Оценка точности и полноты поиска определялась следующим образом:

$$
P=\frac{n}{N}, R=\frac{n}{M},
$$

где $\boldsymbol{P}$ - точность (рис. 9), $\boldsymbol{R}$ полнота (рис. 10), $\boldsymbol{n}$ - число

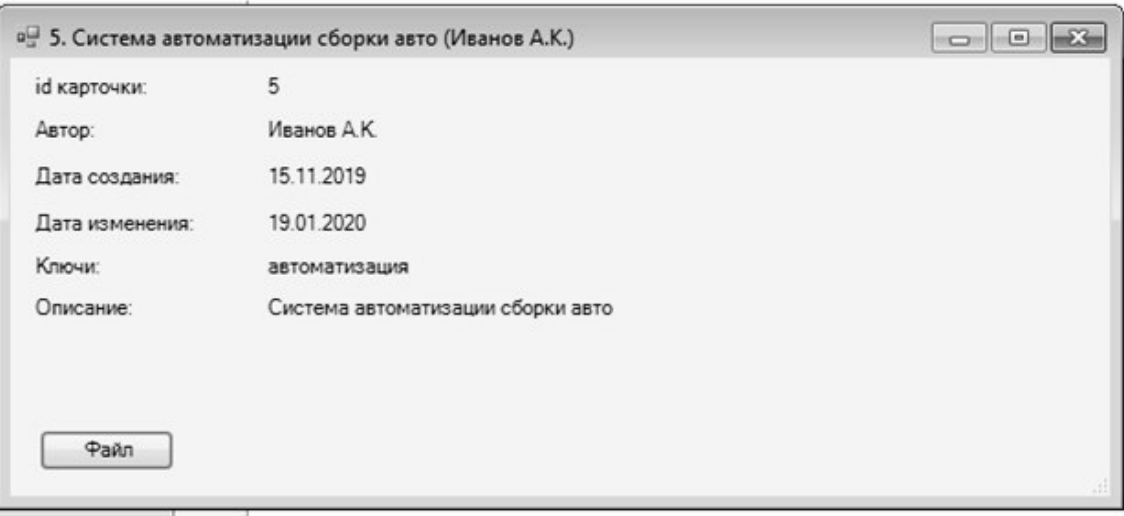

Рис. 7. Окно просмотра записи

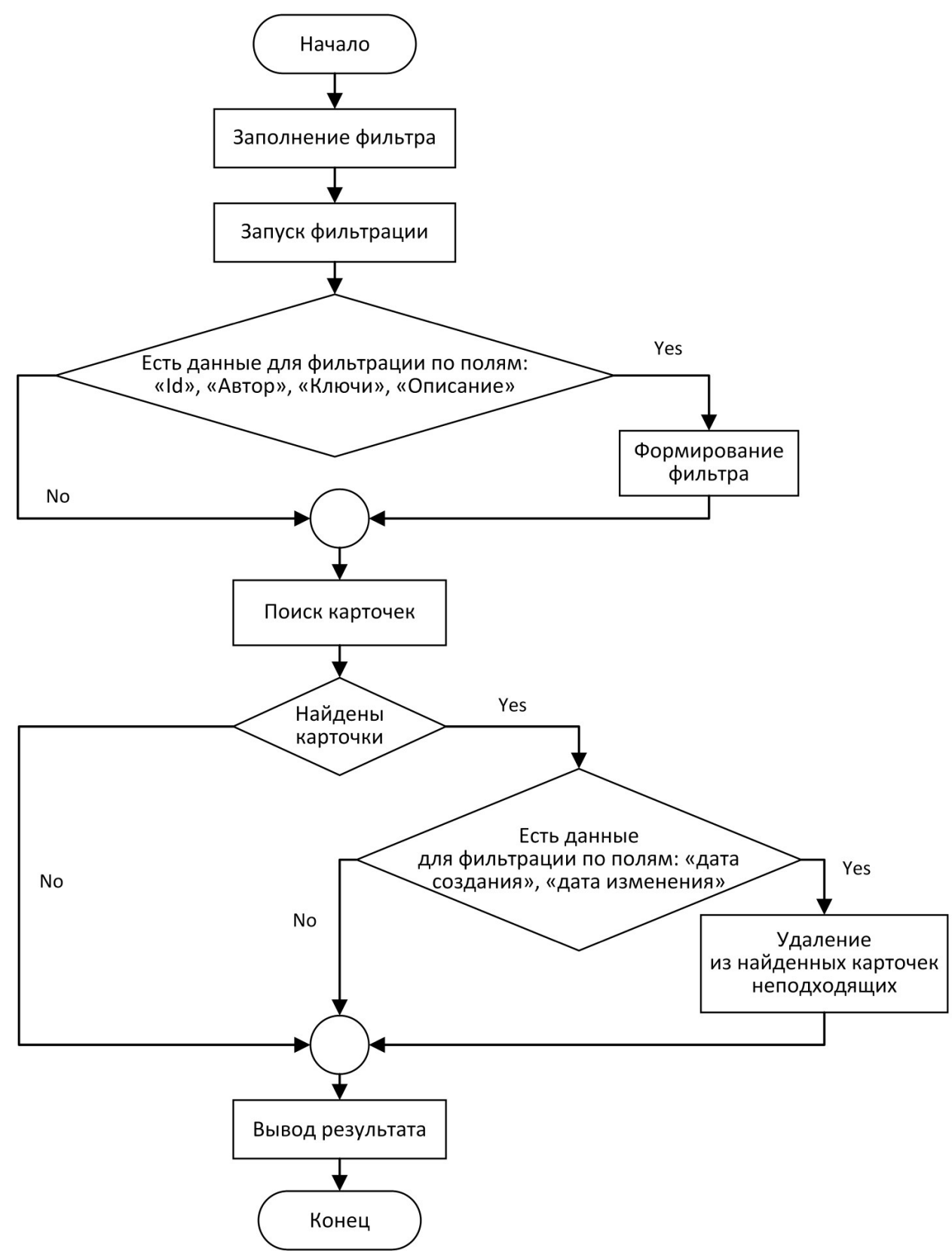

Рис. 8. Поиск прецедентов 
найденных релевантных прецедентов, $\boldsymbol{N}$ - общее число найденных прецедентов, $\boldsymbol{M}$ - общее число релевантных прецедентов.

В ходе эксперимента были проанализированы два фактора, влияющих на эффективность поиска, а именно: погруженность пользователя в информацию, которая хранится базе, и алгоритм поиска.

Для оценки эффекта от уровня погруженности в эксперименте приняли участие проектировщики их трех групп: инженер по знаниям (в т. ч. автор базы прецедентов по AC), эксперт в области АС, студент.

В эксперименте сравнивались три алгоритма поиска:

- поиск только по одному текстовому полю;

- несколько полей для поиска по конкретным параметрам;

- добавление поиска по диапазону дат.

Входе проведения эксперимента были получены результаты, которые изображены на рисунках 9 и 10. После анализа результатов были сделаны следующие выводы:

1. В случае поиска по одному полю значение показателя полноты поиска высокое, но при этом точность низкая. Эта закономерность прослеживается у всех участников эксперимента.

2. При осуществлении поиска по нескольким полям значение полноты уменьшается, но растет точность. Эффект для участников экспериментов отличается. У автора значение полноты уменьшилось на 22\%, у эксперта на $33 \%$, а у студента - на 66\%. Такие значения связаны с общим ухудшением полученных результатов. При этом значение точности у автора выросло на 1 и 75\%, у эксперта - на $160 \%$, а у студента - на $300 \%$. Наименьшая потеря в значении полноты наблюдается у автора, так как он имеет полное представление о данных, которые хранятся в полях базы данных и в онтологии проекта. Эксперт имеет полное представление об онтологии и недостаточно полное представление о данных, которые хранятся в полях базы данных. Студент не владеет полной информацией об онтологии проекта, но полученный эффект у него самый большой из-за того, что изначально значение точности у него было низкое.

3. Добавление полей поиска по диапазону дат оказало эффект только для автора базы опыта - точность увеличилась на 22\%. Это связано с тем, что он знает временные рамки развития базы опыта и владеет информацией о сроках решения задач. У оставшихся пользователей программного комплекса не наблюдается эффекта от добавления диапазона дат. Это связано с тем, что они не использовали поиск по данному полю.

Так как данный инструмент делался в первую очередь для создания персональной базы опыта проектировщика, то самым эффективным вариантом оказалась фильтрация по нескольким полям, с добавлением поиска по диапазону дат.

\section{ЗАКЛЮЧЕНИЕ}

В статье описан очередной шаг в развитии потенциала инструментария OWnWIQA, помогающего принимать проектировщикам более осознанные и обоснованные решения на этапе концептуального проектирования АC. В реализации этого шага совершенствование инструментария интерпретируется как проект, осуществляемый с помощью средств, уже встроенных в комплекс WIQA, среди которых принципиальное место занимает псевдокодовое программирование.

Одним из важнейших ресурсов любого специалиста является его собственный опыт и опыт других проектировщиков. В отсутствие опыта для решения производственных задач используется интуиция. При этом верные решения, основанные на интуиции, формируются также на основе предыдущего опыта из смежной области. Обучение человека в любой области осуществляется через предоставление образов предметов и понятий, поиск общего между ними, построение абстракций разного уровня, поэтому новые единицы опыта должны формироваться на основе схожести с уже известными.

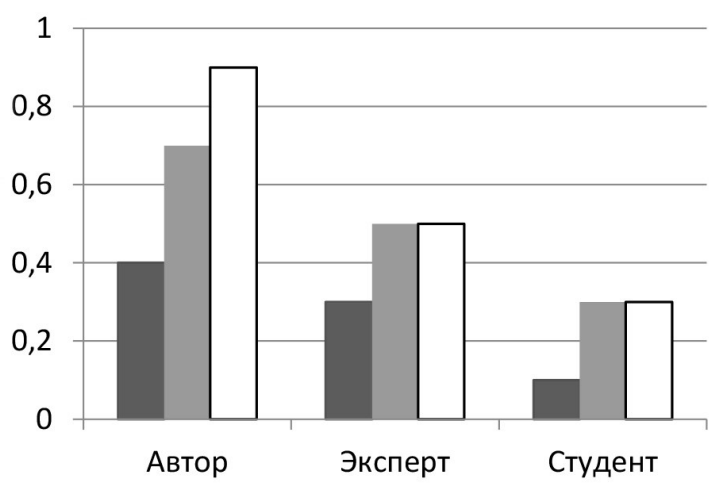

пПоиск по одному полю

Несколько полей

口Диапазон дат

Рис. 9. Исследование точности

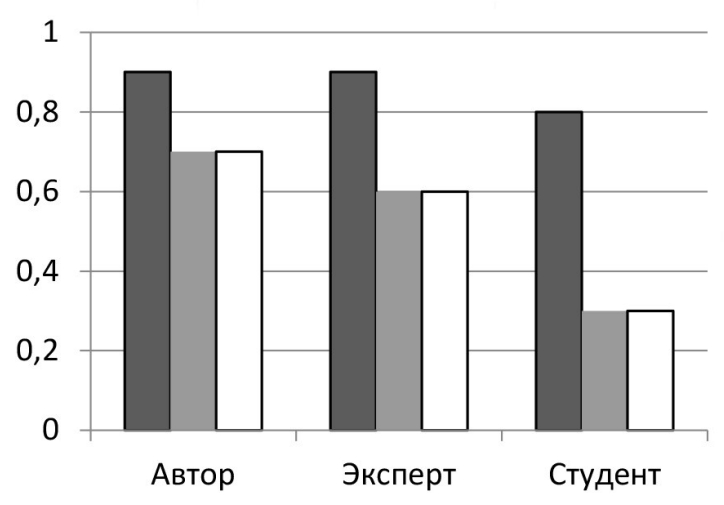

口Поиск по одному полю

- Несколько полей

口Диапазон дат

Рис. 10. Исследование полноты 
Рассмотренные в статье программные средства формирования базы опыта содержат средства для поиска по уже имеющимся карточкам прецедентов для реализации принципа подобия в процессе формирования базы прецедентов.

Полученная структура и каталогизация будут учтены при дальнейшем развитии комплекса программных средств для формирования персональной опыта проектировщика путем автоматического формирования связей между карточками прецедентов, что позволит быстрее находить схожие проекты и проверенные решения с целью повышения эффективности разработок АС, интенсивно использующих ПО.

\section{СПИСОК ЛИТЕРАТУРЫ}

1. The Standish Group report $83.9 \%$ of IT projects partially or completely fail. URL: https://www.opendoorerp.com/ the-standish-group-report-83-9-of-it-projects-partially-orcompletely-fail/ (дата обращения: 24.10.2020).

2. Managing for the sustained success of an organization - A quality management, ISO 9004:2009. URL: http://www.iso.org/iso/catalogue_ detail?csnumber=41014 (дата обращения: 18.09.2020).

3. Гришин А.А. Командная работа: роль проектировщика на каждом этапе разработки сайта. URL: https:// cmsmagazine.ru/journal/items-teamwork-the-role-of-thedesigner/ (дата обращения: 20.10.2020).

4. Маклаев В.А., Перцев А.А., Соснин П.И. Подход к построению и использованию персонифицированной модели проектировщика // Автоматизация процессов управления. 2013. № 4 (34). С. 42-49.

5. Соснин П.И. Вопросно-ответное программирование человеко-компьютерной деятельности. Ульяновск : УлГТУ, 2010. 240 с.

6. Ушаков Д.Н. Толковый словарь современного русского языка, М. : «Аделант», 2014. 800 с.

7. Scott W. Ambler, Mark Lines. Disciplined Agile Delivery: A Practitioner's Guide to Agile Software Delivery in the Enterprise. IBM Press, 2012.

8. Basili A.V., Lindvall M., Costa P. Implementing the experience factory concepts as a set of experience bases // In Proc. of the 13-th International Conference on Software Engineering \& Knowledge Engineering, 2001. pp. 102-109.

9. Ras E., Rech J., Weber S. Knowledge services for experience factories // In Proc. of the 5th Conference on Professional Knowledge Management, 2009. pp. 232-241.

10. Маклаев В.А., Соснин П.И. Создание и использование автоматизированной базы опыта проектной организации. Ульяновск : УлГТУ, 2012. 360 с.

11. Соснин П.И., Маклаев В.А. Формирование и использование вопросно-ответных сетей в проектировании автоматизированных систем // Автоматизация процессов управления. 2017. № 4 (50). С. 75-84.

12. Рис Э. Бизнес с нуля: Метод Lean Startup для быстрого тестирования идей и выбора бизнес-модели / пер. с англ. 5-е изд. М. : Альпина Паблишер, 2016. 255 с.

\section{REFERENCES}

1. The Standish Group Report $83.9 \%$ of IT Projects Partially or Completely fail. Available at: https://www. opendoorerp.com/the-standish-group-report-83-9-of-itprojects-partially-or-completely-fail/ (accessed 24.10.2020).

2. ISO 9004:2009. Managing for the Sustained Success of an Organization - A Quality Management. Available at: http://www.iso.org/iso/catalogue_detail?csnumber=41014 (accessed 18.09.2020).

3. Grishin A.A. Komandnaia rabota: rol proektirovshchika na kazhdom etape razrabotki saita [Team Work: A Designer Role at Every Web-Site Development Stage]. Available at: https://cmsmagazine.ru/journal/itemsteamwork-the-role-of-the-designer/ (accessed 20.10.2020).

4. Maklaev V.A., Pertsev A.A., Sosnin P.I. Podkhod k postroeniiu i ispolzovaniiu personifitsirovannoi modeli proektirovshchika [An Approach to a Personalized Designer Model Generation and Use]. Avtomatizatsiia protsessov upravleniia [Automation of Control Processes], 2013, no. 4 (34), pp. 42-49.

5. Sosnin P.I. Voprosno-otvetnoe programmirovanie cheloveko-komputernoi deiatelnosti [Question-Answer Programming the Human-Computer Interaction]. Ulyanovsk, UISTU Publ., 2010. 240 p.

6. Ushakov D.N. Tolkovyi slovar sovremennogo russkogo iazyka [Modern Russian Dictionary]. Moscow, Adelant Publ., 2014. 800 p.

7. Scott W. Ambler, Mark Lines. Disciplined Agile Delivery: A Practitioner's Guide to Agile Software Delivery in the Enterprise. IBM Press, 2012.

8. Basili A.V., Lindvall M., Costa P. Implementing the Experience Factory Concepts as a Set of Experience Bases. In Proc. of the 13-th International Conference on Software Engineering \& Knowledge Engineering. 2001, pp. 102-109.

9. Ras E., Rech J., Weber S. Knowledge Services for Experience Factories. In Proc. of the 5th Conference on Professional Knowledge Management. 2009, pp. 232-241.

10. Maklaev V.A., Sosnin P.I. Sozdanie i ispolzovanie avtomatizirovannoi bazy opyta proektnoi organizatsii [Creation and Application of Automated Experience Base of Software Design Organization]. Ulyanovsk, UISTU Publ., 2012. 360 p.

11. Sosnin P.I., Maklaev V.A. Formirovanie i ispolzovanie voprosno-otvetnykh setei $\mathrm{v}$ proektirovanii avtomatizirovannykh sistem [Generation and Application of Question-Answer Networks in Designing Automated Systems]. Avtomatizatsiia protsessov upravleniia [Automation of Control Processes], 2017, no. 4 (50), pp. 75-84.

12. Ris E. Biznes s nulia: Metod Lean Startup dlia bystrogo testirovaniia idei i vybora biznes-modeli. Per. s angl. 5-e izd. [The Lean Startup Method for High-Speed Ideas Testing and Business Model Selection. 5th edition transl. from Engl.]. Moscow, Alpina Publ., 2016. 255 p. 